\title{
Pneumococcal antigen detection in bronchoalveolar lavage fluid from patients with pneumonia
}

\author{
Patricio Jiménez, Mónica Meneses, Francisco Saldías, Maira Velásquez
}

\begin{abstract}
Background - Pneumococcal pneumonia can be diagnosed by the detection of capsular antigen in sputum, serum, pleural fluid, or urine using countercurrent immunoelectrophoresis and latex agglutination. In addition, quantitative cultures of bronchoalveolar lavage (BAL) fluid are also reliable for establishing the aetiology of pneumonia. This study investigated the value of rapid detection of pneumococcal antigen in BAL fluid from patients with pneumonia.
\end{abstract}

Methods - Pneumococcal antigen was detected by countercurrent immunoelectrophoresis and latex agglutination. Patients were grouped according to BAL quantitative culture results into pneumococcal pneumonia $(n=24)$, other known aetiology $(n=18), \quad$ and unknown aetiology $(n=17)$. Thirteen patients with interstitial lung disease and without pneumonia served as a control group.

Results - In patients with pneumococcal pneumonia, antigen was detected by countercurrent immunoelectrophoresis in 50\% and by latex agglutination in $54 \%$ of cases. In patients with pneumonia of unknown aetiology pneumococcal antigen was detected by latex agglutination in $53 \%$ of cases. Antigen was not detected in patients with pneumonia of other known aetiology or in control patients, yielding a specificity of $100 \%$.

Conclusions - In patients with pneumococcal pneumonia requiring fibreoptic bronchoscopy detection of pneumococcal antigen in BAL fluid may rapidly and accurately confirm the aetiology. Furthermore, in nearly half the cases of pneumonia of unknown aetiology antigen can be detected, suggesting that Streptococcus pneumoniae is a major causative agent in such patients.

(Thorax 1994;49:872-874)

The establishment of an aetiological agent in community acquired pneumonia remains an unresolved problem. Despite extensive investigations the aetiology of pneumonia remains undetermined in up to $50 \%$ of patients. ${ }^{1-4}$ Diagnostic investigations are usually limited to examination of sputum by Gram stain and culture, and sometimes blood cultures. Unfortunately sputum is not available in one third of patients early in the illness ${ }^{35}$ and, when it is obtained, the results may not establish the cause. Furthermore, the use of antimicrobials before culture often precludes the growth of some organisms. Even when pathogens are isolated results may not be available for 48 hours or longer after hospital admission.

To overcome these deficiencies methods of detecting capsular antigens by using countercurrent immunoelectrophoresis ${ }^{67}$ and latex agglutination $^{8}$ have been developed. These techniques are not affected by prior antibiotic treatment and provide rapid results. Sputum, serum, and urine have been studied with the aim of detecting pneumococcal antigens. ${ }^{57-10}$ The results have been variable depending on the population studied and the kind of specimens used.

Bronchoalveolar lavage (BAL) has been used as an additional technique to fibreoptic bronchoscopy for investigating the aetiology of pneumonia. There is little information about the reliability of pneumococcal antigen detection in BAL fluid from patients with pneumonia. We have shown previously that Streptococcus pneumoniae was the main agent in our patients. ${ }^{11}$ We therefore investigated the detection of Str pneumoniae antigen in the BAL fluid from patients with pneumonia and compared the findings with conventional culture techniques.

\section{Methods}

PATIENTS

Patients with community acquired pneumonia were selected for inclusion into two studies designed to investigate the value of BAL in the diagnosis of bacterial pneumonia, and the evaluation of non-resolving pneumonia. A group of patients with community acquired pneumonia underwent fibreoptic bronchoscopy with BAL before starting antibiotics to ascertain the aetiology of pneumonia. ${ }^{11}$ Community acquired pneumonia was defined as an acute febrile illness with transient shadows on the chest radiograph. A further group of patients with non-resolving pneumonia was also studied with fibreoptic bronchoscopy and BAL. Non-resolving pneumonia was defined as the persistence of fever, clinical, and radiographic signs of pneumonia beyond five days of antibiotic treatment. ${ }^{12}$

Patients were subsequently reclassified into three groups. (1) Pneumococcal pneumonia $(n=24)$ was diagnosed if blood cultures yielded Str pneumoniae or cultures of BAL fluid yielded $\geqslant 10^{3} \mathrm{cfu} / \mathrm{ml}$ Str pneumoniae. This group included 18 men and six women of mean age 40 
(range 18-88) years. Of these patients 10 were alcoholics, eight were smokers, three had chronic obstructive pulmonary disease (COPD), and one each had diabetes, asthma, gallstones, chronic renal failure, and long term use of corticosteroids. Two patients had had influenza in the previous two weeks and seven patients had no associated illness. Blood cultures were set up on admission and, when possible, a sputum sample was obtained for Gram stain and culture. All underwent fibreoptic bronchoscopy and BAL to obtain samples for quantitative culture. ${ }^{11}$ (2) Pneumonia of other known aetiology occurred in 18 patients with isolation of a non-pneumococcal pathogen at counts $\geqslant 10^{3} \mathrm{cfu} / \mathrm{ml}$ from BAL fluid. Of these patients four had Haemophilus influenzae pneumonia (three had COPD) and one had Haemophilus parainfluenzae pneumonia. The remaining patients had non-resolving pneumonia and the organisms recovered were Staphylococcus aureus in four, Gram negative bacilli in six (one each of $H$ influenzae, Hafnia alvei, Klebsiella pneumoniae, Acinetobacter calcoaceticus, Enterobacter sp, and Kpneumoniae plus $A$ calcoaceticus), Candida sp in two, and Staph epidermidis in one. All were initially undiagnosed and were receiving antibiotic treatment when fibreoptic bronchoscopy and BAL was carried out. (3) Pneumonia of unknown aetiology occurred in 17 patients in whom no positive microbiological results were obtained including blood culture. Three of these patients did not expectorate, in five patients sputum culture yielded normal flora, and the remaining nine had non-resolving pneumonia with negative diagnostic tests on admission.

A control group of 13 patients with interstitial lung disease underwent fibreoptic bronchoscopy as part of their routine investigations. These patients had neither clinical nor radiographic signs of pneumonia, and BAL fluid cultures were negative or yielded normal flora with low colony counts.

All patients gave informed consent.

\section{BRONCHOSCOPY}

The bronchoscope was wedged in the most affected site according to the chest radiograph and $180 \mathrm{ml}$ of sterile normal saline was instilled and gently aspirated. ${ }^{11}$ The recovered fluid was pooled and quantitative cultures were performed under aerobic and anaerobic conditions. ${ }^{1314}$ In addition an aliquot of BAL fluid was stored at $-20^{\circ} \mathrm{C}$ until assayed for pneumococcal antigen.

\section{ANTIGEN DETECTION}

Countercurrent immunoelectrophoresis

Glass slides $(6.9 \times 6.9 \mathrm{~cm})$ were coated with $7 \mathrm{ml}$ of $0.75 \%$ agarose (Merck) in barbitone buffer $0.05 \mathrm{mmol} / \mathrm{l}\left(\mathrm{pH} \mathrm{8.6)} .^{15}\right.$ Two rows of paired wells, each $4 \mathrm{~mm}$ in diameter, were punched immediately before use. Ten $\mu$ l antiserum (Omniserum, Statens Seruminstitut, Copenhagen, Denmark) was placed on the anode side and $10 \mu \mathrm{l}$ BAL fluid was placed in a paired well. Slides were arranged in an electrophoretic chamber containing $500 \mathrm{ml}$ barbitone buffer ( $\mathrm{pH} 8 \cdot 6$ ) and a constant current of $20 \mathrm{~mA}$ was applied for 45 minutes at room temperature. Slides were assessed immediately after electrophoresis for visible bands. A positive control was prepared by extracting a sonicated suspension of Str pneumoniae with ethanol and redissolving the precipitate in saline phosphate buffer.

\section{Latex agglutination}

The Wellcogen ZL22 kit (Murex Diagnostics, Dartford, UK) was used following the manufacturer's instructions. BAL fluid samples were thawed and immersed in boiling water for five minutes; $20 \mu \mathrm{l} \mathrm{BAL}$ fluid was mixed with reagents on an appropriate card. Agglutination was assessed with the naked eye three minutes after mixing.

\section{STATISTICAL ANALYSIS}

Sensitivity was defined as the number of positive countercurrent immunoelectrophoresis or latex agglutination tests divided by the number of tests in confirmed Str pneumoniae pneumonia. Specificity was defined as the number of negative countercurrent immunoelectrophoresis or latex agglutination tests divided by the number of tests in pneumonia of other known aetiology. ${ }^{16}$

\section{Results}

Streptococcus pneumoniae was detected in sputum by Gram staining and culture in five and eight cases respectively of the 18 patients with pneumococcal pneumonia. In the remaining six patients sputum could not be obtained. Blood cultures yielded Str pneumoniae in six of the 24 patients and serum countercurrent immunoelectrophoresis was positive in only one patient, whereas pneumococcal antigen was detected in BAL fluid in 12 patients by countercurrent immunoelectrophoresis and in 13 patients was detected by latex agglutination, yielding a sensitivity of $50 \%$ and $54 \%$ respectively (table).

In none of the patients with pneumonia caused by other known aetiological agents was pneumococcal antigen detected, yielding a specificity of $100 \%$ for pneumococcal infection. Furthermore, in all 13 patients with interstitial lung disease studied as controls negative results were obtained.

In patients with pneumonia of unknown aetiology pneumococcal antigen was detected in BAL fluid from four of eight cases by countercurrent electrophoresis and in nine of 17 cases by latex agglutination. All these patients had negative results by sputum Gram stain and culture, blood culture, and serum countercurrent electrophoresis.

\section{Discussion}

Various methods have been used to detect pneumococcal antigen in serum, sputum, urine, and pleural fluid from patients with pneumonia, though there are few data on the value of 
Results of antigen detection in BAL fluid and conventional cultures from patients with community acquired pneumonia*

\begin{tabular}{|c|c|c|c|c|c|c|}
\hline \multirow[t]{3}{*}{ Diagnosis } & \multicolumn{6}{|c|}{ No. of positive cases/no. tested (\%) } \\
\hline & \multirow{2}{*}{$\begin{array}{l}\text { Sputum } \\
\text { Gram stain }\end{array}$} & \multirow{2}{*}{$\begin{array}{l}\text { Sputum } \\
\text { culture }\end{array}$} & \multirow{2}{*}{$\begin{array}{l}\text { Blood } \\
\text { culture }\end{array}$} & \multirow{2}{*}{$\begin{array}{l}\text { Serum } \\
\text { CIE }\end{array}$} & \multicolumn{2}{|l|}{$B A L$ antigen } \\
\hline & & & & & CIE & $L A$ \\
\hline Pneumococcal pneumonia $(n=24)$ & $5 / 18(28)$ & $8 / 18(44)$ & $6 / 24(25)$ & $1 / 24$ & $12 / 24(50)$ & $13 / 24(54)$ \\
\hline Pneumonia of other known aetiology $(n=18)$ & & & $0 / 18$ & 0.5 & $0 / 5$ & $0: 18$ \\
\hline Pneumonia of unknown aetiology $(n=17)$ & $0 / 14$ & $0 / 14$ & $0 / 17$ & $0 / 5$ & $4 / 8(50)$ & $9 / 17(53)$ \\
\hline
\end{tabular}

$\mathrm{CIE}=$ countercurrent immunoelectrophoresis; $\mathrm{LA}=$ latex agglutination

* Patients classified according to BAL quantitative cultures.

detection in BAL fluid. Our results show that pneumococcal capsular antigen can be detected in BAL fluid from patients with pneumonia, and that latex agglutination and countercurrent immunoelectrophoresis have a comparable sensitivity. We suggest that latex agglutination is the technique of choice because it is faster to perform and requires less equipment than countercurrent immunoelectrophoresis.

We have previously established the reliability of quantitative cultures of BAL fluid for the aetiological diagnosis of pneumonia in patients with moderately severe infection, ${ }^{11}$ although detection of pneumococcal antigen occurred in only $54 \%$ of patients with established pneumococcal infection, a sensitivity lower than in sputum or pleural fluid. ${ }^{817}$ We do not know the reasons for this discrepancy. There was no relation between the number of colony forming units and the results of latex agglutination. Performing antigen detection immediately after BAL and not in frozen samples as was the case in this study might improve antigen detection.

In our series pneumococcal antigen detection in BAL fluid was highly specific, with no detection of antigen in patients with pneumonia of other known aetiology, or in the control subjects with interstitial lung disease. The specificity obtained was better than that reported for sputum ${ }^{8}$ and may result from contamination of sputum with oropharyngeal secretions causing false positive results.

In up to $50 \%$ of patients with community acquired pneumonia the microbial aetiology is never determined despite extensive studies. It has been postulated that other agents causing pneumonia remain to be discovered. ${ }^{18} \mathrm{An}$ alternative suggestion is that most patients with pneumonia of unknown aetiology have pneumococcal pneumonia. ${ }^{519}$ Our results are in accord with the latter explanation since 53\% of our patients with pneumonia of unknown aetiology had pneumococcal antigen detected in BAL fluid - a similar proportion to that of patients with pneumococcal pneumonia.

The clinical implications of our results suggest that patients with pneumonia first undergo non-invasive investigations - for example, blood culture and sputum Gram staining and culture. If patients do not produce sputum, as happens in up to half the cases, the severity of illness needs to be determined. In mild cases an antibiotic for the pathogen considered the most likely aetiological agent may be prescribed on an empirical basis. In more severe cases fibreoptic bronchoscopy with quantitative cultures of BAL fluid and antigen detection may be of value for establishing pneumococcal aetiology with accuracy. Antigen detection in BAL fluid may be of value, not only for the patients with pneumococcal pneumonia and positive cultures, but also for more than half the patients whose cultures will not be diagnostic.

The authors are indebted to Dr Juan Carlos Bertoglio and 1)r Sonia Arriagada for reviewing the manuscript.

This study was supported by the Comision Nacional de This study was supported by the Comision Nacional do Investigacion Cientifica y Tecnológica (FONDECYT Grant
No. 955/91) and the Dirección de Investigación y Desarrollo, No. 955/91) and the Dirección de Investigación y D
Universidad Austral de Chile (DID Grant S-93-()3).

1 Bates JH, Campbell D, Barron AL, McCracken CA, Morgan $\mathrm{PN}$, Moses EB, et al. Microbial etiology of acute pneumonia in hospitalized patients. Chest 1992;101:1005-12.

2 Woodhead MA, MacFarlane JT, McCracken JS, Rose DH, Finch RG. Prospective study of the aetiology and outcome of pneumonia in the community. Lancet 1987; i:671 4

3 Levy M, Dromer F, Brion N, Leturdu F, Carbon C. Community-acquired pneumonia. Importance of initial noninvasive bacteriologic and radiographic investigations. Chest 1988;92:43-8.

4 Marrie TJ, Grayston JT, Wang SP, Kuo CC. Pneumonia associated with the TWAR strain of Chlamydia. Ann Intern Med 1987; 106:507-11.

5 Research Committee of British Thoracic Society and Public Health Laboratory Service. Community-acquired pneumonia in adults in British hospitals 1982-1983. a survey of aetiology, mortality, prognostic factors and outcome. $Q$ f Med 1987;239:195 -220.

6 Coonrod JD, Rytel MW. Detection of type-specific pneumococcal antigens by counterimmunoelectrophoresis. I. Methodology and immunologic properties of pneumococcal antigen. F Lab Clin Med 1973;81:770) 7

7 Tugwell P, Greenwood BM. Pneumococcal antigen in lobar pneumonia. F Clin Pathol 1975;28:118-23.

8 Boersma WG, Löwenberg A, Holloway Y, Kuttschrütter H, Snijder JAM, Koëter GH. Pneumococcal capsular antigen detection and pneumococcal serology in patients with community acquired pneumonia. Thorax 1991;46:902-6.

9 Ortquist A, Jonsson I, Kalin M, Krook A. Comparison of three methods for detection of pneumococcal antigen in sputum of patients with community-acquired pneumonia. sputum of patients with community-acquired
Eur $\mathcal{7}$ Clin Microbiol Infect Dis 1989;8:956-61.

10 Coonrod JD, Drennan DP. Pneumococcal pneumonia: capsular antigenemia and antibody responses. Ann Interm Med 1976;84:254-60

11 Jiménez P, Saldias F, Meneses M, Silva ME, Wilson MG, Otth L. Diagnostic fiberoptic bronchoscopy in patients with community-acquired pneumonia. Comparison between bronchoalveolar lavage and telescoping plugged catheter cultures. Chest 1993;103:1023-7.

12 Feinsilver SH, Fein AM, Niederman MS, Schultz DE, Faegenburg DH. Utility of fiberoptic bronchoscopy in nonresolving pneumonia. Chest 1990;98:1322-6.

13 Winterbauer RH, Hutchinson JF, Reinhardt GN, Sumida SE, Deardew B, Thomas CA, et al. The use of quantitative cultures and antibody coating of bacteria to diagnose cultures and antibody coating of bacteria to diagnose bacterial pneumonia by fiberopti
Respir Dis 1983;128:98-103.

14 Buchanan RE, Gibbons NE. Bergey's manual of determinative bacteriology. 8th ed. Baltimore: Williams \& Wilkins, 1984. 5 Anhalt JP, Kenny GE, Rytel MW. Detection of microbial antigens by counterimmunoelectrophoresis. In: Galvan $\mathrm{TL}$, ed. Cumulative techniques and procedures in clinical microbiology, Cumitech 8, Washington DC: American Society for Microbiology, 1978:1-11.

16 Matthews DE, Farewell VT. Using and understanding medical statistics. Basel, New York: Karger, 1985

17 Boersma WG, Löwenberg A, Holloway Y, Kuttschrütter H, Snijder JAM, Koëter GH. Rapid detection of pneumococcal antigen in pleural fluid of patients with community acquired pneumonia. Thorax 1993;48:160-2

18 Fang GD, Fine M, Orloff J, Arisumi D, Yu VL, Kapoor W, et al. New and emerging etiologies for community-acquired pneumonia with implications for therapy. Medicine 1990; 69:307-16.

19 Farr BM, Kaiser DL, Harrison BDW, Connolly CK. Prediction of microbial aetiology at admission to hospital for pneumonia from the presenting clinical features. Thorax 1989;44:1031-5. 